\title{
REVIEW
}

\section{Signal transduction mediated by Bid, a pro-death Bcl-2 family proteins, connects the death receptor and mito- chondria apoptosis pathways}

\author{
YIN XIAO-MING \\ Department of Pathology, University of Pittsburgh School of \\ Medicine, 3550 Terrace Street, Pittsburgh, PA 15261, USA
}

\begin{abstract}
Two major apoptosis pathways have been defined in mammalian cells, the Fas/TNF-R1 death receptor pathway and the mitochondria pathway. The Bcl-2 family proteins consist of both anti-apoptosis and pro- apoptosis members that regulate apoptosis, mainly by controlling the release of cytochrome $\mathrm{c}$ and other mitochondrial apoptotic events. However, death signals mediated by Fas/TNF-R1 receptors can usually activate caspases directly, bypassing the need for mitochondria and escaping the regulation by Bcl-2 family proteins. Bid is a novel pro-apoptosis Bcl-2 family protein that is activated by caspase 8 in response to Fas/TNF-R1 death receptor signals. Activated Bid is translocated to mitochondria and induces cytochrome c release, which in turn activates downstream caspases. Such a connection between the two apoptosis pathways could be important for induction of apoptosis in certain types of cells and responsible for the pathogenesis of a number of human diseases.
\end{abstract}

Key words: Apoptosis, Bcl-2 family proteins, Bid, Fas, TNF

Programmed cell death (PCD) is an active process of cellular self-destruction with distinctive morphological and biochemical features[1]. PCD is indispensable in the development and maintenance of homeostasis within all multicellular organisms. The molecu-

* Correspondence to: Xiao Ming Yin, MD, PhD.

Tel: 1-412-648-8436; Fax: 1-412-383-9594; E-mail: xmyin@pitt.edu 
Bid mediated mitochondria apoptosis pathway

lar machinery of PCD is also important for the regulation of homeostasis during adulthood, which is important for the control of neoplasia and autoimmunity. Understanding how apoptosis occurs in different situations will help to understand the pathogenesis of a number of human diseases and therefore provide clues to the treatment.

\section{Genetic and biochemical pathways of apoptosis}

A genetic pathway that regulates PCD has been defined and appears to be conserved from the nematode $C$. elegans to mammals[2]. Several key groups of molecules have been identified. Caspases are the main effector molecules that are activated and/or regulated by molecules that are responsive to death or survival signals[3]. The substrates of caspases include caspases themselves and other cellular proteins. The activation of one caspase by another constitutes the caspase cascade that not only amplifies the process but also transmits the signals from one compartment to another within cells. Based on the relative substrate specificity, the structure, and the function in the caspase cascade, caspases that participate in apoptosis can be categorized into upstream initiator caspases, such as caspase 8 or 9 , and downstream effector caspases, such as caspase 3 or 7[3]. Caspases are activated or inactivated through a series of intracellular steps, or pathways, in response to death or survival signals, which are subject to multiple regulations. There are two major apoptotic pathways defined in mammalian cells, the death receptor pathway and the mitochondria pathway (Fig 1).

The death receptor pathway is initiated at the cell surface through the Fas/TNF-R1 family protein[4]. Ligation of Fas either by its ligand, FasL, or by its agonistic antibodies triggers the homotrimeric association of the receptors. The clustering of the death domains in the intracellular portion of the receptors recruits the adapter molecule, FADD, which then recruits pro-caspase 8 . Activation of pro-caspase 8 through selfcleavage leads to a series of downstream events, including activation of pro-caspase 3 , cleavage of multiple caspase substrates and induction of mitochondrial damages. In a similar fashion, binding of TNF to TNF-R1 results in the trimerization of the receptor and recruitment of TRADD, which is followed by a similar reaction seen in the activation of Fas receptors. The known difference is that TRADD also recruits other molecules, such as TRAF2, which actually activates a protective pathway through the transcription factors NF- $\kappa$ B. Thus in many types of cells, TNF induces cell death only in the presence of transcriptional inhibitors.

There are, however, a large number of death stimuli that do not seem dependent on the death receptor pathway. Instead, the death signals are transmitted to mitochondria through unique intracellular signaling pathways, where release of cytochrome $\mathrm{c}$ is induced[5]. Cytochrome c activates Apaf-1, in the presence of dATP, which in turn activates pro-caspase 9 . Activated caspase 9 can then cleave downstream effector caspases. Mitochondria apoptosis pathway is involved in many types of cell death induced by stress signals, such as irradiation, DNA-damaging drugs, hormone or growth factor 
withdrawal[5]. It has to be pointed out that cytochrome $\mathrm{c}$ release and caspase activation may not be the only effects caused by the various insults on mitochondria. Others include mitochondrial deplorization and free radical generation.

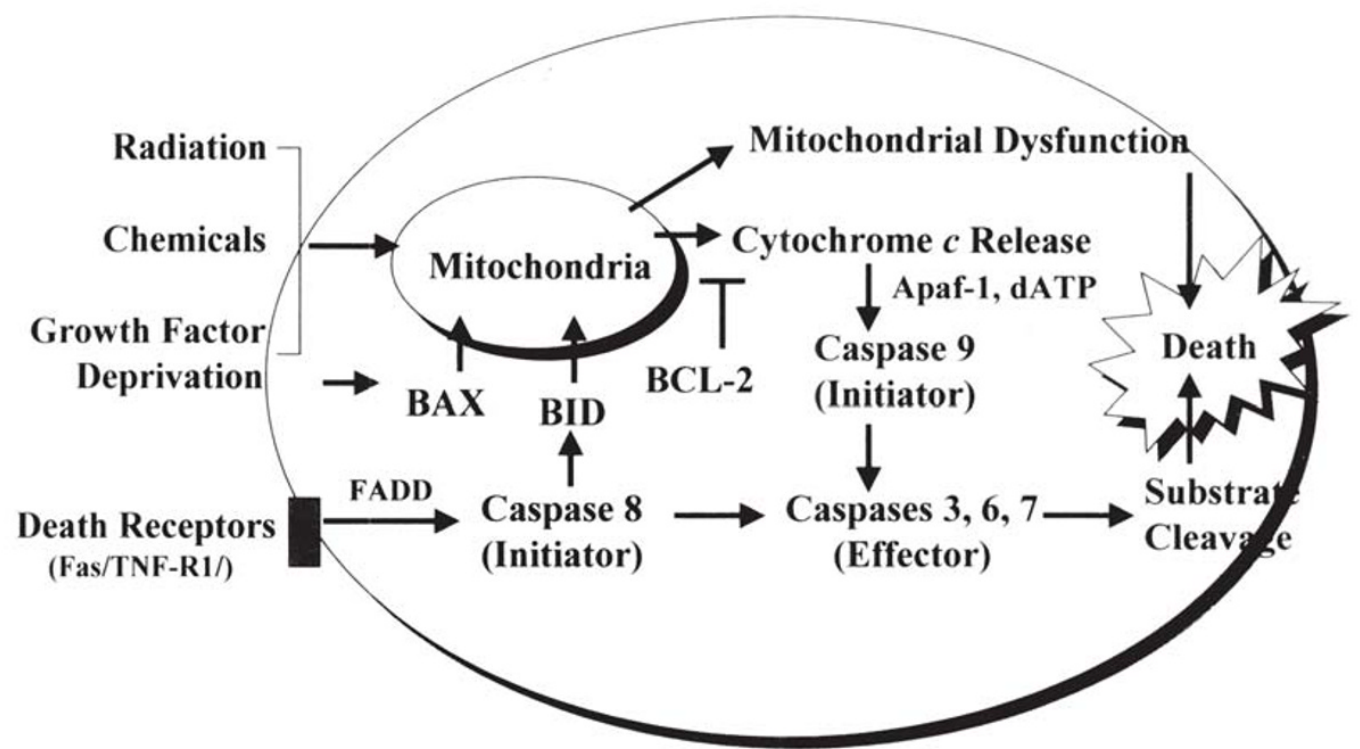

Fig 1. Two major apoptosis pathways are present in mammalian cells. One is mediated by mitochondria, where cytochrome $\mathrm{c}$ is released in response to death stimuli, which in turn activates Apaf-1 and caspases. Mitochondria may mediate other apoptotic events (mitochondrial dysfunction) that are contributory to final cell demise, such as opening of permeability transition pore. The mitochondria pathway is subject to the regulation by Bcl-2 family proteins. Death stimuli transmitted through the Fas/TNF-R1 death receptor family are mainly mediated directly by caspase cascades in cytosol. However, in certain types of cells, such as hepatocytes, the effector caspases may not be efficiently activated by caspase 8 and thus the mitochondria pathway mediated by Bid becomes critical. Bid is cleaved by caspase 8 and translocated to mitochondria to induce cytochrome c release. In other types of cells, such as lymphocytes, this mitochondrial branch may also play a role in further enhancing the effects of the cytosolic caspase pathway.

\section{Bcl-2 family proteins regulate apoptosis via the mitochondria pathway}

This family of proteins consists of both death antagonists (BCL-2, BCL-XL etc.) and death agonists (BAX, Bid etc.)[5]. They share structural homology in BH1, 2, 3 and 4 domains, although not all members have all domains. The $\mathrm{BH} 1$ and $\mathrm{BH} 2$ domains of the antagonists are required to heterodimerize with the death agonists and repress cell death. Conversely, the BH3 domain of the death agonists is required for them to heterodimerize with the death antagonists and to promote cell death[5-7]. 
Bid mediated mitochondria apoptosis pathway

It now seems clear that Bcl-2 family proteins regulate apoptosis mainly via the mitochondria pathway. Bcl-2 family proteins can regulate caspase activation through the regulation of cytochrome $\mathrm{c}$ release, which is inhibited by the death antagonists (Bcl-2 or Bcl-xL), and promoted by the death agonists (Bax or Bid)[5]. The molecular natures these effects are still elusive. Toward this end, Bid, a unique BH3-only molecule of the Bcl-2 family protein may provide important clues. Bid was first cloned from a cDNA expression library based on its interaction with Bcl-2 and Bax[7]. The proapoptotic activity of Bid depends on the BH3 domain. Recently, it was found that Bid could be activated by death signals transmitted by the Fas/TNF-R 1 death receptors. Bid could be cleaved by activated caspase 8[8-10]. The appearance of the cleaved p15 product was detected first in cytosol but later, on the mitochondria, where it induced cytochrome c release[8-10]. The fact that cytoplasm of activated cells depleted of Bid by immunoprecipitation was no longer able to induce cytochrome c release indicated that Bid was the only effector molecule downstream of caspase 8 that had such a capability [10]. Functionally, truncated Bid was more potent than the full length one to induce cell death as well as to interact with Bcl-xL[8]. The key question, however, is what is the physiological significance of Bid being activated by Fas/TNF-R1 signaling. To answer this question, we studied an animal model involving bid-deficient mice.

\section{The role of Bid in Fas-mediated hepatocyte apoptosis}

Activation of Fas-mediated apoptosis in vivo can be achieved by a single injection of an agonist anti-Fas antibody. Mice usually die within $6 \mathrm{~h}$ of receiving a lethal dose of anti-Fas antibody, and $\mathrm{Fas}^{l p r} /{ }^{p r}$ mice that do not have functional Fas are completely resistant to this treatment[11]. The major organ affected is the liver and the massive hepatocyte apoptosis is responsible for the animal death[11]. Furthermore, isolated hepatocytes are susceptible to anti-Fas antibody induced apoptosis in vitro, demonstrating the direct cytotoxic effects of Fas activation in this type of cells[12]. caspases$3 / 7$ activities are detected in liver cells of mice injected with anti-Fas antibodies as well as in hepatocytes incubated with anti-Fas in vitro[12]. Furthermore, hepatocytes transgenically over-expressing Bcl-2 are resistant to anti-Fas antibody-induced injury in vivo[13]. These data support the idea that the toxicity of Fas activation in hepatocytes may be mainly mediated via the mitochondria pathway, in that caspase activation is stimulated by the release of cytochrome c, which is inhibited by Bcl-2 or Bcl-xL. We thus hypothesized that it was Bid that was responsible for the induction of cytochrome c release and activation of caspase downstream of Fas activation.

Indeed, a single intravenous injection of anti-Fas antibody induced cleavage of Bid in liver and caused its translocation to mitochondria[10]. To further demonstrate the role of Bid in anti-Fas mediated apoptosis, we studied bid-deficient mice, which were constructed through homologous recombination[14]. These mice were born alive and had no apparent gross development abnormalities. The anti-Fas antibody was given 
intravenously at a dose of $0.25 \mu \mathrm{g} / \mathrm{g}$. Wild type animals were almost universally susceptible to the lethal effect of anti-Fas at this dosage and died within $6 \mathrm{~h}$. The survival rate in the $24 \mathrm{~h}$ period was 1 out 8 animals. Strikingly, bid-/-mice were resistant to this anti-Fas induced mortality, $82 \%$ (9/11) bid-/-mice survived within the same time period. Upon the injection of the anti-Fas antibodies, the livers of wild type animals increased dramatically in weight and the histology showed extensive hepatic cell apoptosis and hemorrhaging necrosis, as reported previously. All these changes were much smaller or absent in the livers of bid-/-mice. DNA fragmentation could be clearly demonstrated in wild type but not in bid-/-livers by TUNEL staining. Isolated biddeficient hepatocytes were also resistant to anti-Fas antibodies in vitro. These data clearly indicate that bid-/- hepatocytes are resistant to anti-Fas induced apoptosis and further suggest that Bid is essential to Fas-mediated hepatocyte apoptosis.

Fas activation normally initiates the cytosolic apoptosis pathway through direct caspase recruitment and activation in cells such as lymphocytes. The much reduced lethality and hepatic apoptosis in bid-/-mice suggested that in hepatocytes, the main apoptotic pathway initiated by Fas activation was in fact the mitochondria pathway mediated by Bid. Indeed, activities of caspase 3 or 7 were detected in wild-type mice receiving anti-Fas antibody treatment, which was hardly detectable in the livers of bid/-mice. In contrast, there were no differences in the activities of the upstream initiator caspase 8 , as analyzed by Western blot and substrate cleavage assay. Correspondingly, the immunostaining signals of cytochrome $\mathrm{c}$ were much diminished in hepatocytes of wild type animals in response to Fas stimulation, implying the release of cytochrome $c$ from mitochondria. But the signals were not changed in bid-/-animals, suggesting that Bid was likely the only molecule transmitting the mitochondrial signals in Fas activation. These finding are consistent with the idea that Bid acts downstream of Fas receptor and caspase 8 , but upstream of cytochrome $\mathrm{c}$ release and effector caspase activation. Moreover, it suggests that in hepatocytes, the mitochondria pathway mediated by Bid is much more important than the cytosolic pathway in determining caspase activation and the cell fate in response to anti-Fas antibody treatment.

\section{The role of Bid in TNF-R1 initiated hepatocyte apoptosis}

Since Bid is critical for Fas-signaling induced apoptosis in hepatocytes, it could be equally important to TNF-R1 mediated hepatocyte death, where the signaling pathway is similar. This notion is supported by the finding that Bid can be cleaved and translocated to mitochondria in response to TNF in a lymphoid cell line[10]. The availability of several murine models of TNF mediated liver injuries makes the test of this hypothesis feasible.

The most commonly used in vivo models of TNF toxicity in mice involve endotoxemiarelated hepatic failures. In one of such models, lipopolysaccharide (LPS) is used to stimulate the production of TNF by macrophages[15]. This treatment, when combined 
Bid mediated mitochondria apoptosis pathway

with a pre-administration of an amino sugar, D-galactosamine (GalN), a liver specific transcriptional inhibitor, causes liver-specific damages. The primary hepatotoxic effect of TNF in this model is the induction of apoptosis of hepatocytes[16], [17] and the activation of caspases can be readily demonstrated[18]. The necrosis and inflammation are also involved, but only at later stage[17].

Although the role of caspases in hepatocyte apoptosis induced by TNF has been firmly established, the signals and cellular components involved in the pathway are just being revealed. We are now testing this hypothesis by examining the response of bid-deficient mice and hepatocytes to TNF. Our preliminary results indicated that Bid was cleaved in mice given LPS and GalN, and that the cleaved p15 Bid was translocated to mitochondria (Zhao et al, manuscript in preparation). Cleavage of Bid was also observed in vitro in cultured hepatocytes treated with TNF and ActD. In both in vivo and in vitro studies, it seems that Bid is very likely an important component in the pathogenesis of TNF-mediated toxicity in hepatocytes.

\section{The implication of the role of Bid in the pathogenesis of diseases of liver and other organs}

The participation of Bid in the Fas/TNF-R1 mediated pathway suggests that it could be one of the key components in the development of a number of human diseases that are related to the Fas/TNF-R1 activation, particularly the liver failure seen in several clinical scenarios. For example, the FasL/Fas interaction has been implicated in the development of viral hepatitis and other types of liver damage[19]. In another scenario, TNF-R1 activation subsequent to septic shock could lead to liver failure as part of the clinical manifestations of endotoxemia. The role of TNF-alpha in the pathogenesis has been clearly demonstrated in patients as well as in animal models[16-18],[20]. The current studies on Bid in animal models suggest that this molecule could be critical for the pathogenesis of these diseases.

Activation of Fas death receptor has also been implicated in human diseases involving other organs, such as pancreas and thyroid. The apoptotic destruction of functional cells in these organs due to either an autoimmune response or injury is thought to be at least in part responsible for the non-obesity diabetes or autoimmune thyroiditis. Whether Bid is essential in the signaling of Fas in these situations has yet to be determined. It has to be cautioned, however, that in some systems, such as lymphoid cells and embryonic fibroblasts, activation of the Fas/TNF-R1 pathway leads to a more direct activation of cytosolic effector caspases, such that the role of Bid and the mitochondria apoptosis pathway may be limited to the amplification of the death signaling [10],[14]. It will be interesting to determine whether other types of cells, such as the bislet cells could be the type of cells in which activation of Fas will rely on the mitochondria pathway, and therefore Bid, to reach the full effects. These studies will thus promote our understanding of the pathogenesis of a number of human diseases, which may 
in turn provide hints for preventional and therapeutic interventions in the future.

\section{REFERENCE}

[1] Kerr JF, AH Wyllie, AR Currie. Apoptosis: a basic biological phenomenon with wide-ranging implications in tissue kinetics. Br J Cancer 1972; 26:239-57.

[2] Horvitz HR, S Shaham, MO Hengartner. The genetics of programmed cell death in the nematode Caenorhabditis elegans. Cold Spring Harb Symp Quant Biol 1994; 59:p.377-85.

[3] Thornberry NA, Y Lazebnik. Caspases: enemies within. Science 1998; 281:1312-6.

[4] Ashkenazi A, VM Dixit. Death receptors: signaling and modulation. Science 1998; 281:1305-8.

[5] Gross A, JM McDonnell, SJ Korsmeyer. BCL-2 family members and the mitochondria in apoptosis. Genes and Development 1999; 13:1899-911.

[6] Yin XM, ZN Oltvai, SJ Korsmeyer. BH1 and BH2 domains of Bcl-2 are required for inhibition of apoptosis and heterodimerization with Bax [see comments]. Nature 1994; 369:321-3.

[7] Wang K, et al. BID: a novel BH3 domain-only death agonist. Genes Dev 1996; 10:2859-69.

[8] Li H, et al. Cleavage of BID by caspase 8 mediates the mitochondrial damage in the Fas pathway of apoptosis. Cell 1998; 94:491-501.

[9] Luo X, et al. Bid, a Bcl2 interacting protein, mediates cytochrome c release from mitochondria in response to activation of cell surface death receptors. Cell 1998; 94:481-90.

[10] Gross A, et al. Caspase Cleaved BID Targets Mitochondria and Is Required for Cytochrome c Release, while BCL-XL Prevents This Release but Not Tumor Necrosis Factor-R1/Fas Death. J Biol Chem 1999; 274:1156-63.

[11] Ogasawara, J et al. Lethal effect of the anti-Fas antibody in mice [published erratum appears in Nature 1993 Oct 7; 365(6446):568]. Nature 1993; 364:806-9.

[12] Jones RA et al. Fas-mediated apoptosis in mouse hepatocytes involves the processing and activation of caspases. Hepatology, 1998; 27:1632-42.

[13] Lacronique V et al. Bcl-2 protects from lethal hepatic apoptosis induced by an anti-Fas antibody in mice. Nat Med 1996; 2:80-6.

[14] Yin XM et al. Bid-deficient mice are resistant to Fas-induced hepatocellular apoptosis. Nature 1999; 400:886-91.

[15] Tiegs GM, Wolter, A Wendel. Tumor necrosis factor is a terminal mediator in galactosamine/ endotoxininduced hepatitis in mice. Biochem Pharmacol 1989; 38:627-31.

[16] Pfeffer $\mathrm{K}$ et al. Mice deficient for the $55 \mathrm{kd}$ tumor necrosis factor receptor are resistant to endotoxic shock, yet succumb to L. monocytogenes infection. Cell 1993; 73:457-67.

[17] Leist $\mathrm{M}$ et al. Tumor necrosis factor-induced hepatocyte apoptosis precedes liver failure in experimental murine shock models. Am J Pathol 1995; 146:1220-34.

[18] Jaeschke H et al. Activation of caspase 3 (CPP32)-like proteases is essential for TNF- alpha- induced hepatic parenchymal cell apoptosis and neutrophil-mediated necrosis in a murine endotoxin shock model. J Immunol 1998; 160:3480-6.

[19] Galle PR et al. Involvement of the CD95 (APO-1/Fas) r eceptor and ligand in liver damage. J Exp Med 1995; 182:1223-30.

[20] Tracey KJ, A Cerami. Tumor necrosis factor: an updated review of its biology. Crit Care Med 1993; 21:S415-22.

Received June-26-2000. Revised July-6-2000. Accept ed July-17-2000. 